\title{
Habilidades digitales y desempeño docente en el área de comunicación de educación secundaria, en tiempos de pandemia
}

\section{Digital skills and teaching performance in the communication area of secondary education, in times of pandemic}

\author{
Luis Alberto Torres Inga ${ }^{1 a}$, León Huamán Ramos², Alison Marisu \\ Amancio Anzuhueldo ${ }^{3}$, Sebastián Sánchez Díaz ${ }^{4}$ \\ Universidad Cesar Vallejo, Lima Este, Perú ${ }^{1234}$ \\ ORCID ID: 0000-0002-9511-1445 \\ ORCID ID: 0000-0002-5156-88572 \\ D ORCID ID: 0000-0002-3066-8592 3 \\ D ORCID ID: 0000-0002-0099-7694
}

Recibido: 27 de mayo de 2021

Aceptado: 20 de setiembre de 2021

\section{Resumen}

El objetivo de esta investigación fue analizar de qué manera las habilidades digitales de los docentes del área de comunicación en secundaria, en tiempos de pandemia, generaron aprendizaje. Se aplicó como metodología el enfoque cualitativo, basado en el modelo interpretativo socio crítico y diseño de la teoría fundamentada, empleando la entrevista semiestructurada con una muestra de tres profesores del área de comunicación, seis alumnos y seis progenitores del tercer y cuarto grados, a través de la matriz apriorística y triangulación. Los resultados muestran que la disposición gubernamental para el trabajo remoto, fue recibida de forma disconforme por la comunidad educativa, ya que no existían condiciones para afrontar una educación remota, cuya separación presencial implicó poner en práctica las habilidades digitales. Se encontraron aprendizajes no muy satisfactorios, a causa que varios estudiantes tenían escaso manejo del celular, señal limitada de internet, otros se subían a los árboles o cerros más elevados de su zona para recibir alguna señal, ni desarrollaban satisfactoriamente las separatas. Por otro lado, los padres de familia expresaron que esta pandemia hizo que se duplicaran sus responsabilidades para con sus hijos. En conclusión, el aprendizaje en el área de comunicación está ligada a las competencias digitales con material disponible del estudiante, caso contrario, el aprendizaje se debilita en una educación a distancia, por lo que es momento de promover retos frente a la alfabetización digital.

${ }^{\mathrm{a} C}$ Correspondencia al autor

E-mail: luito1159@gmail.com 
Palabras clave: habilidades digitales, desempeño, docente, comunicación, informática, educación.

\begin{abstract}
The objective of this research was to analyze how the digital skills of teachers in the communication course in high school generated learning in times of pandemic. The qualitative approach was applied as a methodology, based on the socio-critical interpretive model and grounded theory design, well-founded, using the semi-structured interview with a sample of three teachers from the area of communication, six students and six parents of the third and fourth grades, through the a priori matrix and triangulation. The results show that the government provision for remote work, the educational community received it very dissatisfied, since there were no conditions to face a remote education, whose face-to-face separation implied putting digital skills into practice, with teachers finding themselves at the basic level with step to intermediate, thanks to their self-training and training, achieving not very satisfactory learning, because several students had little use of the cell phone, limited internet, others did not have, some climbed the trees or highest hills in their area, or they were developing the reprints satisfactorily. On the other hand, parents expressed that this pandemic doubled their responsibilities towards their children. In conclusion, learning in the area of communication is linked to digital skills with the student's available material, otherwise, learning is weakened in distance education, so it is time to promote challenges in the face of digital literacy.
\end{abstract}

Keywords: skills, digital, performance, teacher, communication, computing, education.

\title{
Introducción
}

Aunque el mundo ha sufrido en el aspecto social y laboral aun se requieren las habilidades digitales del ser humano (Lujan, 2021). E n consecuencia, la epidemia mundial promovió la educación remota, dejando de lado la educación presencial y la enseñanza expositiva, impulsando una renovación en la construcción pedagógica y soporte didáctico, sin que sea suficiente ser usuario de la tecnología. Por ello, Loor (2020) expresó que las instituciones educativas tienen el desafío de integrar eficazmente las herramientas tecnológicas, iniciando situaciones prósperas de habilidades digitales con metodología que fluya aún más en las zonas rurales, lógicamente bajo una gama de estrategias propuestas por el docente. En su opinión, Figallo (2020) manifestó que cierto número de docentes han desarrollado procesos de aprendizaje con escasa habilidad digital, a fin de prosperar la educación a distancia; en cambio, otros se capacitaron en diversos cursos en línea de escaso tiempo; y el resto tenía desconfianza al enfrentar al aula virtual con estudiantes que no entendían. Su estudio concluye que, si el contexto es difícil entre profesores, la situación no será necesariamente mejor entre los estudiantes. 
Por su parte, Pérez ( 2020) recordó que la inclusión de los seres humanos de cualquier edad en la era informática, ha estimulado a una metamorfosis, la misma que provoca resistencias, por lo que, la comunidad académica y científica deben fortalecer las bondades de la educación virtual, que se vienen implementando desde la niñez; de ahí que Cabrera (2021) reconoce los sustentos doctrinarios de Piaget, que se refieren a la genética del niño y sus actos reflejos de succión, aprensión, manipulación, donde el sujeto-objeto por sí mismo no producen conocimiento, sino que intercede la capacidad volitiva del sujeto en su ambiente y, sobre Vigotsky, enfatizó la relación ineludible de la historia sociocultural del niño con su contexto. Por otro lado, Morales (2021) aludió que la educación cubana tiene como objetivo la necesidad de formar la personalidad de un hombre que responda a los retos de su tiempo e ir desarrollando y potenciando a lo largo de la vida, aplicando metodologías que desarrollen habilidades, no sólo tecnológicas, sino socioemocionales que estimulen un buen desempeño; para ello, Manrique (2021) incidió que es preponderante la influencia de la alfabetización digital en el desempeño docente, especialmente en el uso de las herramientas digitales, ya que la información fluye por diversos medios, de ahí que es de gran necesidad fortalecer la competencia digital de los docentes, a fin de propulsar este proceso de cambio constante.

Aguirre (2020) señala que Ecuador, al igual que el resto del mundo, ha sido dañada por la epidemia, decidiendo también suspender la asistencia presencial y sus actividades en las instituciones, necesidad ineludible para que la comunidad educativa, partiendo del docente, incluyan servicios de mensajes con diversas estrategias comunicativas virtuales, incluyendo separatas pedagógicas. Al respecto, Huamán (2021) explicó que la educación a distancia implica enfrentar con fuerza amenazas de salud, pobreza, desnutrición, por lo que se hace necesario emplear medios y materiales tecnológicos para fortalecer los conocimientos digitales, brindando buena respuesta a la educación remota. En este sentido, el docente es el cimiento de una educación formadora, parte esencial de la modalidad a distancia, quien, a través de dispositivos y herramientas tecnológicas, adapta cambios significativos y experiencias de aprendizaje de manera virtual con sus estudiantes (Azañedo, 2021).

A la luz de estos antecedentes, el objetivo de este estudio es analizar las habilidades digitales de los profesores del área de comunicación en secundaria, que generó aprendizaje en tiempos de pandemia, Posso (2021) contextualizó el propósito, manifestando que la política educativa ecuatoriana, frente a la emergencia saludable incitada por la pandemia ya conocida, 
planteó prevalecer los contenidos en todos los currículos por áreas y aplicación de aprendizaje basado en proyectos visionarios como alternativas metodológicas para la educación remota, basado en la centralidad de aprendizaje del estudiante. En tal efecto, con la actuación y manejo de computadora portátil o de bolsillo con conexión a internet y buena memoria, se puede mejorar la educación tecnológica de manera activa, creativa y eficiente; por ende, un buen aprendizaje se propulsa por un buen desempeño docente (Criollo, 2021). Correlativamente, la pandemia también creó estados de ansiedad, elevado nivel de incertidumbres, riesgos, decepciones e impactos emocionales, por lo que fue necesario diseñar opciones de resolución de problemas, a fin de tratar la tensión en casa, trabajo, estudio, resguardando medianamente una buena salubridad y mejores condiciones de vida (Burgos, 2020).

\section{Metodología}

Esta investigación se realizó bajo el enfoque cualitativo, basado en el paradigma interpretativo socio-crítico, partiendo el estudio con recopilar de manera organizativa la información mediante la observación y autorreflexión de los actores educativos in situ, lenguaje coloquial e interpretativo. Para esto, se emplearon, muestras, croquis, innovación y nuevas teorías en medio de la pandemia, frente a una educación remota (Schenke, 2020). Y, referente al diseño de investigación, se aplicó la metodología de la teoría fundamentada que tiene sustento en las ciencias sociales, la cual se inmiscuye en ella al ser humano en relación con su comunidad educativa, quienes participaron con diversos procedimientos en la recolección de datos en forma abierta en el mismo campo de estudios.

$\mathrm{Al}$ igual que en la entrevista en profundidad o semiestructurada, y a fin de captar diversos puntos de vista de los sujetos implicados, se mantuvo el propósito de vigorizar el fenómeno social investigado, considerándolo como un sistema de descubrimiento científico (Bonilla, 2016). Por su parte, Cerrón (2019) refirió que la participación social en lugares originarios vinculados a su entorno social, ha tenido buenos resultados en las actividades emprendidas en grupos humanos, transfiriendo y construyendo conocimientos que, a partir de sus debilidades, se corrigieron, modificaron, superaron y renovaron sus propósitos en bienestar común.

Cisterna (2005) planteó epistemológicamente la acción del investigador que sostiene su acción en una racionalidad hermenéutica, expresada operacionalmente en los llamados métodos 
cualitativos, plasmados en la entrevista semiestructurada, la cual reconstruye creativamente el proceso de interpretación abordando, estudiando, analizando y construyendo conocimientos en base a la matriz de proceso de categorización y subcategorización apriorística, centradas al objetivo general como propósito global y a los objetivos específicos operacionalizados a ello, distinguiéndose las categorías con su unidad temática. En las subcategorías se detallan dichos campos temáticos en micro aspectos, teniendo en cuenta su reconstrucción creativa en el proceso de la categorización y triangulación, la cual se validó con los conocimientos, luego de la aplicación de los instrumentos recopiladores de la información objetiva y subjetiva y su proceso de interpretación; teniendo en cuenta las premisas, los supuestos y los ejes temáticos en base al objeto de estudio. 


\section{Tabla 1}

Matriz de proceso de categorización y subcategorización apriorística

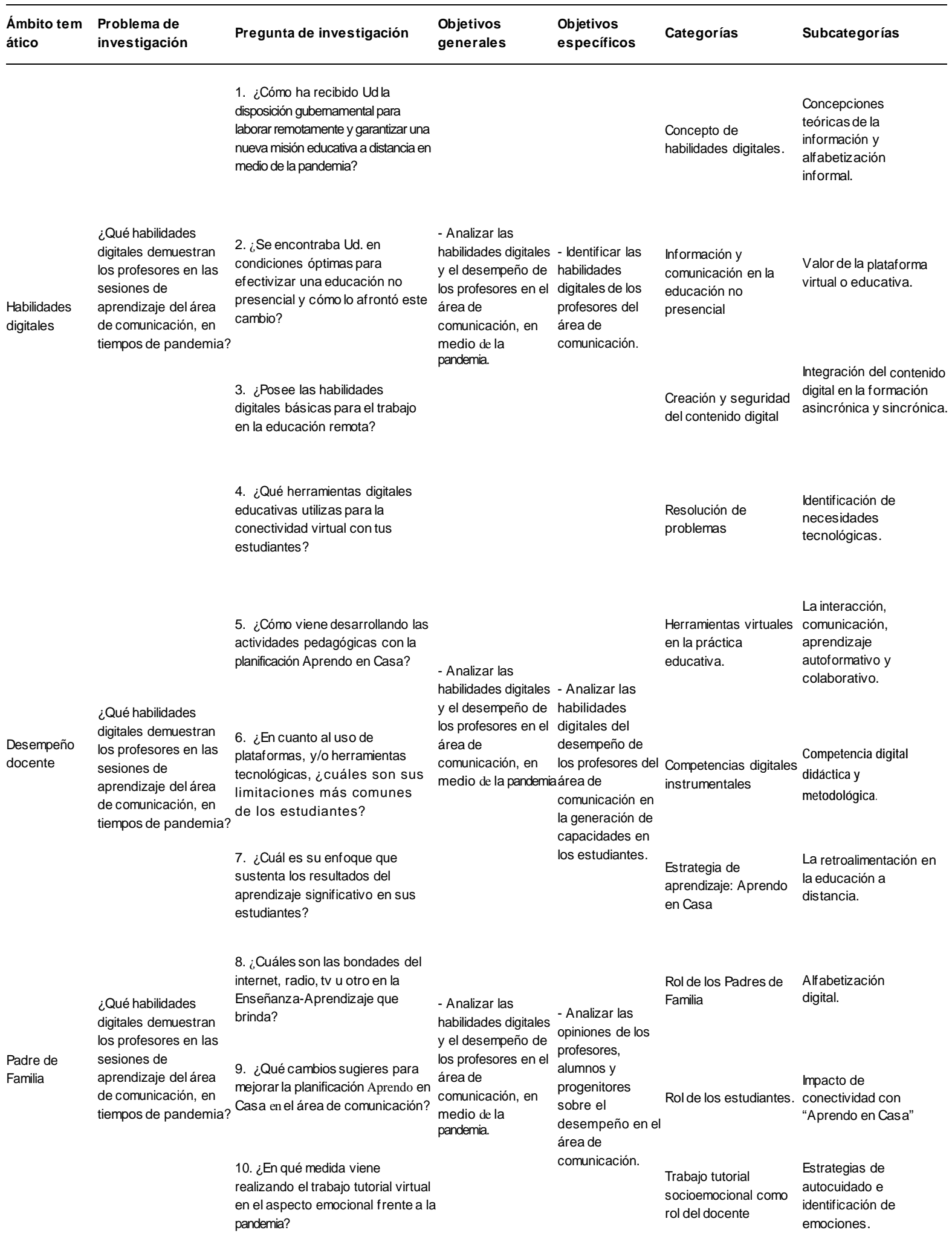

NOTA: Adaptado del estudio de triangulación, como métodos de validación del conocimiento de investigación cualitativa (Cisterna, 2005). 
El campo de estudio fue la Institución Educativa “San Cristóbal,” ubicada en el distrito de Huancavelica, de estrato social media-baja, que brinda servicios educativos a varones y mujeres entre 11 a 16 años que provienen de lugares aledaños en su mayoría y otros de la ruralidad andina. Fue provechoso hacer uso de la triangulación como técnica y herramienta pujante en función de las dimensiones de las variables; para ello se aplicó la entrevista semiestructurada en la muestra de tres profesores del área de comunicación, seis alumnos y seis progenitores del tercer y cuarto grados en medio de la pandemia. En este caso, se hizo la consulta por escrito, solicitando su permiso a través del consentimiento informado, a fin de ser parte del proceso de investigación. Asimismo, se le informó que se mantendría en anonimato y confidencialidad de la información. Por ello, se cruzó y contrastó las diez interrogantes planteadas de manera sincera con diversidad de antecedentes para cada estamento como trabajo de campo, construyendo nuevos conocimientos del objeto en estudio en base a los objetivos, categorías y subcategorías planteadas (Charres, 2018). 


\title{
Tabla 2
}

\section{Cuadro de análisis de información}

\begin{tabular}{cccc}
\hline Subcategorías & $\begin{array}{c}\text { Preguntas al estamento de } \\
\text { docentes del área de } \\
\text { comunicación }\end{array}$ & $\begin{array}{c}\text { Preguntas al estamento de } \\
\text { estudiantes }\end{array}$ & $\begin{array}{c}\text { Preguntas al estamento de } \\
\text { padres de familia }\end{array}$ \\
\hline
\end{tabular}

\section{1. ¿Cómo ha recibido usted la disposición \\ Concepciones teóricas de gubemamental para laborar remotamentey la información y \\ alfabetización informal \\ distancia en medio de la pandemia?}

\begin{abstract}
Valor de la plataforma virtual o educativa.
\end{abstract}

- Integración del contenido
digital en la formación
asincrónica y sincrónica.

Integración del contenido digital en la formación asincrónica y sincrónica.

\begin{abstract}
La interacción, comunicación, aprendizaje auto formativo y colaborativo.
\end{abstract}

Competencia digital didáctica y metodológica.

La retroalimentación en la educación a distancia.

Alfabetización digital

Impacto de conectividad con "Aprendo en Casa"

Estrategias de autocuidado e identificación de emociones.

2. ¿Se encontraba Ud. en condiciones óptimas para efectivizar una educación no presencial y cómo lo afrontó este cambio?

3. ¿Posee las destrezas manuales elementales, intermedias o avanzadas hacia la labor educativa remota?

4. ¿Qué herramientas digitales educativas utilizas para la conectividad virtual con tus estudiantes?

5. ¿Cómo viene desarrollando las actividades pedagógicas en lo que concierne a la planificación Aprendo en Casa?

6. ¿En cuanto al uso de plataformas, y/o herramientas tecnológicas, ¿cuáles son sus limitaciones más comunes de los estudiantes?

7. ¿Cuál es su enfoque que sustenta los efectos de sus enseñanzas significativas en sus alumnos?

8. ¿Cuáles son las bondades del internet, radio, tv $u$ otro en la Enseñanza-Aprendizaje que brinda?

9. ¿Qué cambios sugieres para mejorar la planificación Aprendo en Casa en el área de comunicación?

10. ¿En qué medida viene realizando el trabajo tutorial virtual en el aspecto emocional frente a la pandemia? clases virtuales en el área de
¿Cuál fue tu reacción, en cuánto recibiste la noticia que la educación ya no sería presencial, sino remota durante el año escolar 2020?

¿Cómo ha recibido Ud. emocionalmente la información sobre la educación de su hijo, que ya no sería presencial, sino a distancia el presente año escolar 2020?

¿Te encontrabas preparado para no compartir el mismo espacio físico con tus compañeros y docentes?

¿Se encontraba Ud. y miembros de la familia preparados para atender la educación de su hijo en casa?

¿Posee las destrezas manuales elementales hacia el trabajo en la educación remota?

¿Qué dificultades observó que tiene su hijo(a) para el trabajo remoto en el área de comunicación?

¿Cómo recibes las sesiones de aprendizaje de tu profesor del Área de Comunicación?

¿Cómo observas la participación de tu hijo en las actividades de aprendizaje del Área de Comunicación?
¿Cómo viene participando en las actividades pedagógicas en el área de comunicación?

¿Cuáles son las limitaciones más comunes del profesor del área de comunicación?

¿Qué limitaciones existen en casa para garantizar una mejor educación de su hijo?

¿Cuál es su apreciación sobre los aprendizajes alcanzados en el área de comunicación en esta educación remota?

¿Qué bondades aprecias de las comunicación?

¿Cómo vienen apoyando a su hijo(a) en casa, frente a la educación remota?

¿Qué opinión tiene sobre el ejercicio docente en el área de comunicación?

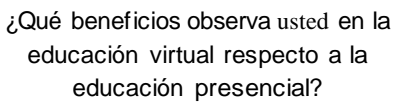

¿Qué cambios es necesario implementar para el trabajo remoto por parte de los docentes?

¿Qué cambios es necesario implementar parar el trabajo remoto por parte de los docentes?

¿Has recibido orientaciones por parte del profesor cuando algunos de tus compañeros mostraban tener problemas, durante la pandemia?
¿En qué medida cumplió el trabajo tutorial virtual el docente en el proceso de la educación remota? 
Asimismo, Cisterna (2005) resaltó que la validez y confiabilidad del conocimiento descansa en última instancia en el rigor del investigador; por ello, se procesó la triangulación de la información por cada estamento, y entre las diversas formas de información, aplicando la conversación oral virtual semiestructurada, a través de video llamadas, Whatsapp, Facebook, Google Meet, entre otros; orientando buen uso de horarios, previsión de buenas condiciones en su domicilio, interrogantes para los tres estamentos, uso de las técnicas e instrumentos de recolección de datos, recopilación de manera organizada de las entrevistas grabadas para su procesamiento, a través de la matriz apriorística y triangulación ascendente de los estamentos, cuya catalogación de información lo plasmamos como desenlace del trabajo de investigación.

En este caso, Orozco (2020) acentuó que el modelo o paradigma interpretativo hermenéutico, tiene como aliado a la tradición verbal como técnica de exploración del paradigma cualitativo, la cual permite la conversación en profundidad y prácticas vivenciales, recopilando datos de manera organizada. Por ello, Fuster (2019) consideró como perspectiva paradigmática a la hermenéutica, en su dimensión cualitativa; en vista que reconstruye tipologías aplicando el pensamiento creativo bajo experiencias vividas, las cuales tienen que ser interpretadas para aprender pedagógicamente, relacionándola indudablemente con la epistemología, como parte de la filosofía. 
Tabla 3

Cuadro de análisis de información

\begin{tabular}{|c|c|c|c|c|}
\hline $\begin{array}{l}\text { CAMPO } \\
\text { TEMATICO }\end{array}$ & DEFINICIÓN CONCEPTUAL & CATEGORÍAS & SUBCATEGORÍAS & FRASES FRECUENTES \\
\hline \multirow[t]{4}{*}{$\begin{array}{l}\text { Habilidades } \\
\text { Digitales }\end{array}$} & $\begin{array}{l}\text { COLAS (2019) } \\
\text { La persona y su contexto innegable } \\
\text { sostienen al enfoque sociocultural, } \\
\text { quienes promueven aprendizaje en ese } \\
\text { contacto. }\end{array}$ & $\begin{array}{l}\text { Concepto de } \\
\text { habilidades digitales. }\end{array}$ & $\begin{array}{l}\text { - Concepciones teóricas de } \\
\text { la información y } \\
\text { alfabetización informal. }\end{array}$ & $\begin{array}{l}\text { - Educación virtual. } \\
\text { - Cuarentena. } \\
\text { - Trabajo remoto. } \\
\text { - Servicio educativo }\end{array}$ \\
\hline & $\begin{array}{l}\text { Estos ejes articulan como un traslado } \\
\text { de evolución de la habilidad manual } \\
\text { tecnológica en el encuentro de } \\
\text { maestro-pupilo. }\end{array}$ & $\begin{array}{l}\text { Versión e } \\
\text { interpretación de la } \\
\text { virtualidad. }\end{array}$ & $\begin{array}{l}\text { - Plataforma virtual o } \\
\text { educativa. }\end{array}$ & $\begin{array}{l}\text {-Respeto el aprendizaje } \\
\text { digital. } \\
\text { - Plataforma. } \\
\text { - Institucional. } \\
\text { - Normas de convivencia. } \\
\text { - Confinamiento. }\end{array}$ \\
\hline & $\begin{array}{l}\text { Consecuentemente a ello, en esa } \\
\text { relación social genera capacidades en } \\
\text { el uso de los medios y materiales } \\
\text { resaltantes tecnológicos. }\end{array}$ & $\begin{array}{l}\text { Creación y seguridad } \\
\text { del contenido digital. }\end{array}$ & $\begin{array}{l}\text { - Integración del contenido } \\
\text { digital en la formación } \\
\text { asincrónica y sincrónica. }\end{array}$ & $\begin{array}{l}\text { - Nivel básico. } \\
\text { - Manejo del aplicativo } \\
\text { Whatsap. } \\
\text { - Cuarentena. } \\
\text { - Conectividad. }\end{array}$ \\
\hline & & $\begin{array}{l}\text { Resolución de } \\
\text { problemas }\end{array}$ & $\begin{array}{l}\text { - Identificación de } \\
\text { necesidades tecnológicas. }\end{array}$ & $\begin{array}{l}\text { - Capacitación. } \\
\text { - Internet, computadora y } \\
\text { laptop. }\end{array}$ \\
\hline \multirow[t]{3}{*}{$\begin{array}{l}\text { Desempeño } \\
\text { Docente }\end{array}$} & $\begin{array}{l}\text { CÓNDOR (2019) La calidad del } \\
\text { desempeño docente debe centrarse en } \\
\text { primera instancia en el ser humano, en } \\
\text { la reflexión permanente de como } \\
\text { enseñar. }\end{array}$ & $\begin{array}{l}\text { Herramientas } \\
\text { virtuales en la } \\
\text { práctica educativa. }\end{array}$ & $\begin{array}{l}\text { La interacción, } \\
\text { comunicación, aprendizaje } \\
\text { autoformativo y } \\
\text { colaborativo. }\end{array}$ & $\begin{array}{l}\text { - Trabajo pedagógico virtual. } \\
\text { - Enfoque por competencias. } \\
\text { - Aprendizaje autónomo. } \\
\text { - Sesiones de aprendizajes } \\
\text { virtual. }\end{array}$ \\
\hline & & $\begin{array}{l}\text { Competencias } \\
\text { digitales } \\
\text { instrumentales }\end{array}$ & $\begin{array}{l}\text { Competencia digital } \\
\text { didáctica y metodológicas. }\end{array}$ & $\begin{array}{l}\text { - Cobertura en tiempo real. } \\
\text { - Planificación curricular. } \\
\text { - Adquisición de ordenadores. }\end{array}$ \\
\hline & $\begin{array}{l}\text { Para mejorarla buena práctica } \\
\text { pedagógica innegablemente tiene que } \\
\text { existir un compromiso personal y } \\
\text { social capaz de romper las barreras. }\end{array}$ & $\begin{array}{l}\text { Estrategia de } \\
\text { aprendizaje: Aprendo } \\
\text { en casa }\end{array}$ & $\begin{array}{l}\text { La retroalimentación en la } \\
\text { educación a distancia }\end{array}$ & $\begin{array}{l}\text { - Contextualizar aprendo en } \\
\text { casa. } \\
\text { - Trabajo a distancia } \\
\text { - Oportunidad. } \\
\text { - Responsabilidad. }\end{array}$ \\
\hline \multirow[t]{3}{*}{$\begin{array}{l}\text { Padre de familia } \\
\text { en la gestión } \\
\text { escolar }\end{array}$} & $\begin{array}{l}\text { SUCARI (2019) Hoy más que nunca } \\
\text { la situación en que vivimos es } \\
\text { reflexiva; a su vez comprensible } \\
\text { frente a la reactivación económica y }\end{array}$ & $\begin{array}{l}\text { Rol de los padres de } \\
\text { familia }\end{array}$ & Alfabetización digital. & $\begin{array}{l}\text {-Apoyo digital. } \\
\text {-Seguimiento en tareas. } \\
\text {-Conectividad con los } \\
\text { docentes }\end{array}$ \\
\hline & $\begin{array}{l}\text { social de las familias, en salud y } \\
\text { educación; por ello, se hace presente } \\
\text { los deberes y derechos como }\end{array}$ & Rol de estudiantes & $\begin{array}{l}\text { Conectividad Aprendo en } \\
\text { Casa }\end{array}$ & $\begin{array}{l}\text { - Portafolio. } \\
\text { - Textos auto instructivos. } \\
\text { - Tv - Radio }\end{array}$ \\
\hline & $\begin{array}{l}\text { ciudadanos, las cuales les permiten } \\
\text { participar activamente a las familias } \\
\text { en el sistema educativo que bien } \\
\text { puede ser a través de la APAFA. }\end{array}$ & $\begin{array}{l}\text { Trabajo tutorial socio } \\
\text { emoción al docente. }\end{array}$ & $\begin{array}{l}\text { Estrategias de autocuidado e } \\
\text { identificación de emociones. }\end{array}$ & $\begin{array}{l}\text { - Orientación. } \\
\text { - Apoyo con sugerencias. } \\
\text { - Confianza. } \\
\text { - Normas de convivencia. }\end{array}$ \\
\hline
\end{tabular}

Nota: Tomado del resultado de la entrevista semiestructurada a docentes, estudiantes y padres de familia

\section{Resultados}

En cuanto al dispositivo gubernamental viceministerial peruano, que planteó avalar una nueva misión educativa a distancia en medio de la pandemia mundial, los tres estamentos educativos recibieron la noticia con bastante preocupación y disconformidad, en vista que no estuvieron en buenas condiciones óptimas de afrontar una educación a distancia. Los estudiantes eran procedentes en un $70 \%$ de zona rural, la señal de internet, radio y TV eran escasas, a las ocho 
de la mañana se perdía la conectividad, por ejemplo. En consecuencia, la comunidad educativa realizó un trabajo colegiado, buscando medios o mecanismos para tener acceso con los estudiantes, frente a la separación física; la cual implicó enfrentar y adecuarse a la problemática, acudiendo a diversas estrategias como el uso de redes sociales, a sabiendas que eran adolescentes; pese a no encontrarse capacitados en competencias informáticas y no contar con su implementación. Los docentes expresaron conocer algunos entornos virtuales de aprendizaje, gracias a su autocapacitación y capacitación, ubicándose en el nivel básico y otros ingresando al nivel intermedio con el internet, correo electrónico, almacenamiento de archivos en la nube, Whatsapp Web, Messenger, Zoom, Google Meet, uso de portales educativos con la estrategia “Aprendo en casa," insertando en sus sesiones de aprendizaje en el Whatsapp de preferencia y experimentalmente en el Zoom o Meet con foros, diapositivas, fotografías, videos, exposiciones atendiendo dudas y preguntas, retos, trabajos autónomos, en pares, retroalimentación, producción de textos y portafolio del estudiante.

Por su parte, los padres de familia valoraron el desempeño docente, reconociendo que esta pandemia duplicó sus responsabilidades más que todos los años y los estudiantes expresaron que los docentes promovieron aprendizaje con lecturas, producción de textos regionales relacionados a la COVID-19, comunicación biunívoca entre pares que no funcionó en algunos casos, ya que muchos estudiantes no pudieron descargar y/o subir los trabajos encomendados; además, escalaron cerros para conectarse, hubo conflictos por el uso del celular; por ello, los docentes optaron con apoyar con separatas para mejorar el aprendizaje, lo cual también requerían mayor explicación, de ahí que los docentes no concluyeron con el avance curricular. Por consiguiente, los padres expresaron que los docentes se adecuaron a la realidad con organización de su tiempo, manejo digital, planificación de “Aprendo en casa”, etc.; en cambio, los docentes plantearon que dicha estrategia no es mala, sino que hay necesidad de contextualizarlas. 


\section{Tabla 4}

Cuadro de análisis resumido del objetivo de investigación

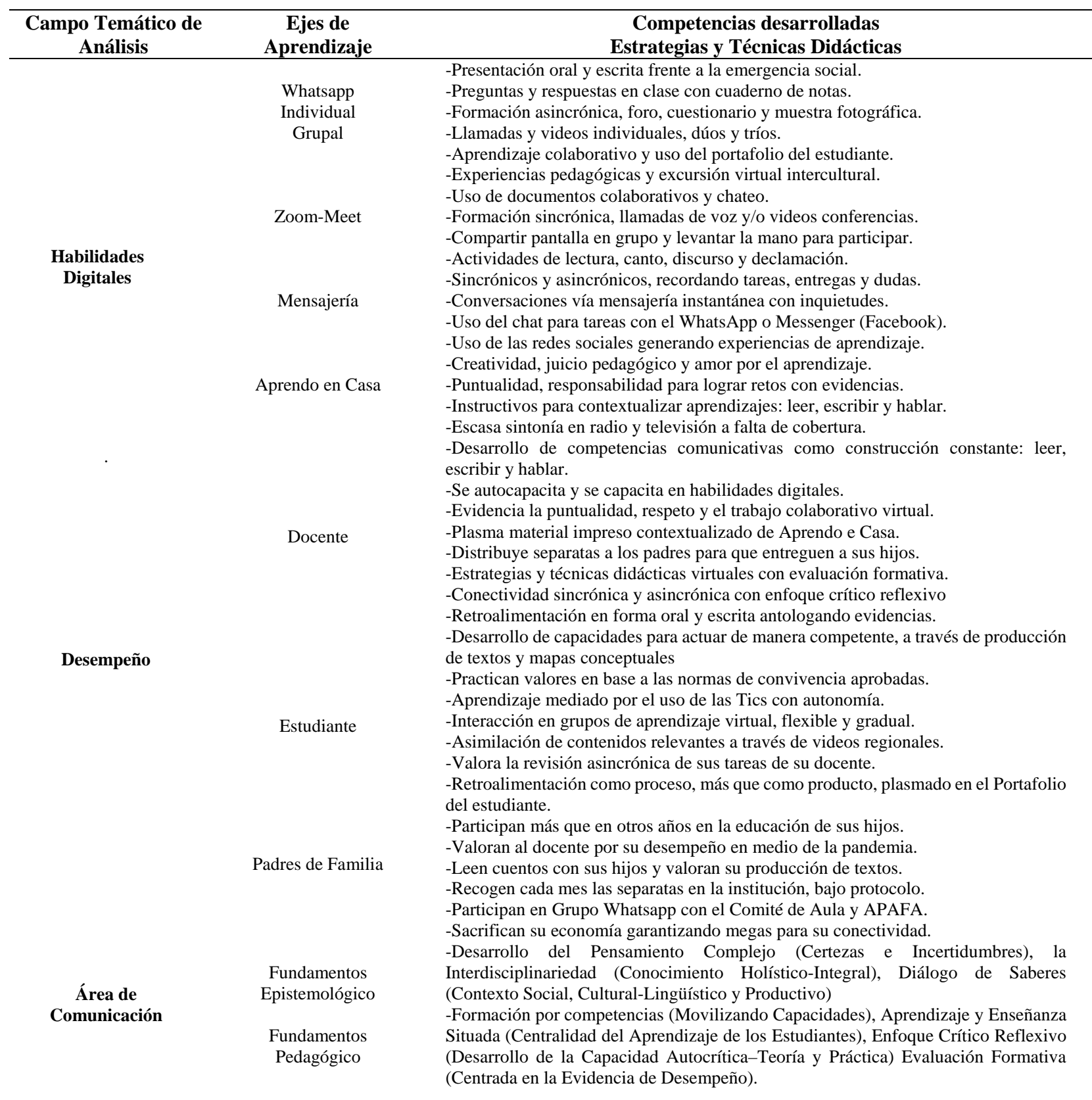

A partir de los hallazgos encontrados afirmamos que, a causa de las disposiciones políticas por orden social, las instituciones educativas asumieron el desafío de integrar herramientas tecnológicas, a fin de garantizar la educación no presencial durante la emergencia social, frente al rebrote de la pandemia. Por ello, Aguirre (2020) coadyuvó con dicha realidad, manifestando que la pandemia mundial también obligó al gobierno ecuatoriano cancelar el encuentro físico con los 
estudiantes en las instituciones educativas. De igual forma Jorna (2021) enfatizó que la pandemia puso a los gobiernos en una crisis que originó inadaptaciones en todos los sectores económicos, políticos y sociales, los cuales enfrentaron el reto de salvar vidas y minimizar su impacto en el enfrentamiento a la COVID-19.

Almida (2021) incidió que la consecución de la pandemia provocó fuertes restricciones e incompatibilidades en las actividades de empresas y personas en la Unión Europea, donde sus efectos no sólo se limitaron al campo de la salud pública, sino que también incluyeron a las dimensiones económica social y educativo. Consecuentemente a ello, las competencias digitales se abrieron paso cada vez más como imprescindibles, a fin de alcanzar una verdadera sociedad de la información y el conocimiento (Diaz, 2021). Análogamente a ello, surgieron factores que contribuyeron en las competencias digitales por parte de los educadores, su capacitación y autocapacitación, más allá de la enseñanza remota, siendo una motivación para fortalecer sus habilidades digitales por parte de los docentes (Beardsley, 2021).

Por otro lado, existe relación de dependencia entre habilidades digitales y desempeño docente para mejorar los aprendizajes, en vista que las tecnologías digitales desempeñaron un papel mediador para con los participantes. Esto sucedió en forma indistinta en el desempeño docente y en el de los estudiantes, cuyo intento de actuación fue alcanzar los objetivos de aprendizaje, mediados por la tecnología e influyéndose mutuamente (Peña, 2021). En nuestra investigación, la tecnología para los estudiantes como señal de internet, radio y TV fueron escasas: a las ocho de la mañana se perdía la conectividad y los estudiantes subían a los cerros o árboles para conseguirla. Por ello, los objetivos de aprendizaje al inicio de la educación remota no eran convincentes, en vista que las brechas digitales se acentuaron por factores de conectividad, dispositivos, apropiación de la tecnología, inadecuada formación docente, entre otros, existiendo la necesidad de potencializar el binomio conectividad y desempeño docente (Anaya, 2021). En tal sentido, bajo el planteamiento de Bulganina (2021), se confirmó que ello guarda relación con los docentes del área de comunicación que se capacitaron en habilidades digitales, estando algunos en el nivel básico y otros en el nivel intermedio. Lo mismo sucedió con los estudiantes, lo cual quizás hubiera sido diverso si la institución educativa contaba con una plataforma virtual.

Por su parte, Moreno (2020) planteó que el docente es persona clave en la actuación educativa, para la transformación de los aprendizajes; para tal efecto, tiene que hacer uso necesariamente de medios y materiales valorables, asimilar experiencias exitosas de otros 
docentes, estar vinculado con la red, determinar su estilo propio de buscador de información. Medina (2021) avaló ello, practicando el apoyo colaborativo en la actuación educativa, la cual es fundamental para el éxito escolar, brindando comunicación, apertura y participación, que son cifras para crear un clima de confianza. García (2021) siendo así, las competencias comunicativas de leer, escribir y hablar se desarrollaron, haciendo uso de las habilidades digitales e inclusión de separatas, respetando normas de convivencia, aplicación de la evaluación formativa y retroalimentación con un enfoque crítico reflexivo, antologando evidencias en el portafolio, producción de textos, fotografías virtuales en el grupo Whatsapp, en algunos casos por intermedio del Zoom o Meet, en vista que la institución no cuenta con una plataforma virtual. Por ello, Valencia (2019) incidió que el desempeño docente radica en la forma como desarrolla el aprendizaje en sus diferentes actividades pedagógicas, dado su planificación curricular, la cual no está vinculado tan directamente con un programa, que le ayude a desarrollar sus labores pedagógicas, sino es en que como hace uso los programas en forma adecuada y que no sólo se requiere tener encendido la máquina tecnológica,

Efectivamente, Marcillo (2021) manifestó que una buena y nueva gestión educativa con objetivos, buen equipo institucional, competencia digital y desarrollo de capacidades a docentes y estudiantes en el marco de una reestructuración curricular basados en el método histórico lógico, con propósitos claros de aprendizaje, promueven buena comprensión lectora, dicción y redacción.

Finalmente, se encontraron las siguientes limitaciones: problemas en la conectividad en docentes, estudiantes, padres de familia y en el proceso de la investigación misma. Por otro lado, los sujetos de estudio fueron renuentes para participar en el proceso-estudiantes procedentes del área rural con escaso acceso a los medios digitales; tal es el caso que varios no contaban con celulares y en algunos casos equipos muy antiguos, siendo otra limitante que no tenían posibilidades económicas para obtener un buen servicio de internet.

\section{Conclusiones}

Se analizaron las habilidades digitales de los profesores del área de comunicación de educación secundaria, en tiempos de pandemia, siendo de preponderancia la generación de aprendizaje de los estudiantes en un 65 \% a través del grupo Whatsapp y en escala menor mediante el Zoom Google, a causa de la limitación de conectividad, lo que determinó distribuir 
en forma gratuita separatas contextualizadas, por parte de sus docentes, también apoyó la estrategia “Aprendo en Casa.”

Del mismo modo, se observó un soporte en la capacitación de un 75 \% de profesores en el automatismo y conducción de recursos y equipos tecnológicos, materiales que eran distantes frente a las capacidades manuales de los alumnos. Asimismo, la participación de los padres fue a través del comité de aula y la asociación de padres de familia, de manera virtual. Asimismo, el contexto en el cual se desarrollaba el proceso enseñanza/aprendizaje generó ansiedad e incertidumbre, lo cual se reflejó en la actividad académica.

\section{Referencias}

Aguirre R. (2020). COVID-19 y la Educación Virtual Ecuatoriana. Investigación Académica 1(2). https://investigacionacademica.com/index.php/revista/article/view/24/39

Almeida, A. (2021). Digital Skills and Their Relevance to the COVID-19 Innovative Responses in the European Union. Polytechnic Institute of Gaya. 13 (3). https://www.igiglobal.com/article/digital-skills-and-their-relevance-to-the-covid-19-innovativeresponses-in-the-european-union/279599

Anaya, T. (2021). Escuelas rurales en el Perú: factores que acentúan las brechas digitales en tiempos de pandemia (COVID- 19) y recomendaciones para reducirlas. Pontificia $\begin{array}{llll}\text { Universidad Católica } & \text { del } & \text { Perú. }\end{array}$ http://revistas.pucp.edu.pe/index.php/educacion/article/view/23568

Beardsley, (2021). Emergency education effects on teacher abilities and motivation to use digital technologies. British Journal of Educational Technology. 52 (4). https://berajournals.onlinelibrary.wiley.com/doi/full/10.1111/bjet.13101

Bocchio, M. (2020). Consecuencias del Cierre de Escuelas por el Covid-19 en la Desigualdades Educativas. Universidad Nacional de Córdoba. https://revistas.uam.es/riejs/search/authors/view?givenName=Mar\%C3\%ADa\%20Cecilia \%20\&familyName=Bocchio\&affiliation=\&country=AR\&authorName=Bocchio\%2C $\% 20$ Mar\%C3\%ADa\%20Cecilia\%20

Burgos Angulo, (2020). La relajación y estrés laboral en el personal docente: una revisión sistemática. Lecturas: Educación Física y Deportes, 25 (266), 109-123. https://www.efdeportes.com/efdeportes/index.php/EFDeportes/article/download/1617/12 50 ?inline $=1$

Cabrera, M. (2021). Desempeño docente en el desarrollo de competencias matemáticas en educación preescolar: una revisión sistemática. Revista científica hacedor, Aiapaec, 1 (5). http://revistas.uss.edu.pe/index.php/HACEDOR/article/view/1619 
Casanova, H. (2020). Educación y pandemia: el futuro que vendrá. Consejo Mexicano de Ciencias Sociales. Casanova. https://www.comecso.com/wpcontent/uploads/2020/08/Covid-12_Casanova.pdf

Cerrón, W. (2019). La investigación cualitativa en educación. Horizonte de la ciencia 9 (17). http://portal.amelica.org/ameli/jatsRepo/59/59717003/59717003.pdf

Charres, H. (2018). Triangulación: Una herramienta adecuada para las investigaciones en las ciencias administrativas y contables. Revista FAECO Sapiens, 1 (1). http://revistas.up.ac.pa/index.php/faeco_sapiens/article/view/575

Cisterna, F. (2005). Categorización y triangulación como procesos de validación del conocimiento en $\quad$ investigación $\quad$ cualitativa. $\quad$ Theoria, 14 (1). https://www.redalyc.org/pdf/299/29900107.pdf

Criollo, S. (2021). Tecnologías de aprendizaje móvil para la educación: beneficios y cuestiones pendientes. Applied sciences 11 (9). https://www.mdpi.com/2076-3417/11/9/4111

Colás B. (2019). El desarrollo de la competencia digital docente desde un enfoque sociocultural. Comunicar 27 (61). https://doi.org/10.3916/C61-2019-02

Cóndor (2019). La evaluación al desempeño directivo y docente como una oportunidad para mejorar la calidad educativa. Revista Cátedra 2 (1). http://200.12.169.32/index.php/CATEDRA/article/view/1436

Diaz, D. (2021). Competencias digitales en el contexto COVID 19: Una mirada desde la

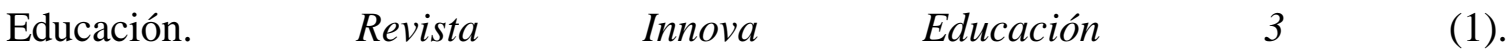
https://www.researchgate.net/publication/348855352_Competencias_digitales_en_el_con texto_COVID_19_una_mirada_desde_la_educacion

Fuster G. (2019). Investigación cualitativa: Método fenomenológico hermenéutico. Propósitos y Representaciones, $\quad 7 \quad$ (1), 201-229. http://www.scielo.org.pe/scielo.php?script=sci_arttext\&pid=S2307-79992019000100010

García, I. (2021). Mapeo de la colaboración de los maestros par el éxito escolar. Taylor \& Francis online.

https://www.tandfonline.com/doi/citedby/10.1080/09243453.2021.1925700?scroll=top\& needAccess $=$ true

Jorna, A. (2021). Gestión de los riesgos sanitarios en el enfrentamiento a la COVID-19 en Cuba. Revista Cubana de Salud Pública $46 \quad$ https://www.scielosp.org/article/rcsp/2020.v46suppl1/e2696/ 
Loor, M. (2020). Uso de las TIC como estrategia de enseñanza para docentes de Educación General Básica en la zona rural. Revista Científica 6 (2) https://dominiodelasciencias.com/ojs/index.php/es/article/view/1246/0

Marcillo, S. (2021). La gestión educativa y su influencia en los nuevos desafíos del desarrollo curricular en la Unidad Educativa Bartolomé Garelli. Universidad Espíritu Santo 6 (1). https://dialnet.unirioja.es/servlet/articulo?codigo $=7897384$

Medina, S. (2021). El aprendizaje cooperativo y sus implicancias en el proceso educativo del siglo XXI. Revista de la Universidad Internacional del Ecuador 30 (58). http://revistas.pucp.edu.pe/index.php/educacion/article/view/23568

Moreno-Guerrero, A., Miaja-Chippirraz, N., Bueno-Pedrero, Ana \& Borrego-Otero, L. (2020), El área de información y alfabetización informacional de la Competencia digital docente en España, Revista electrónica educare 24 (3) https://www.revistas.una.ac.cr/index.php/EDUCARE/article/view/11475/20101

Orozco A. (2020). La Enseñanza aprendizaje de las Ciencias Sociales. Historias de vida de tres maestras de Educación Secundaria de Managua. FAREM-Estelí 34, (4-19). https://www.lamjol.info/index.php/FAREM/article/view/10004

Peñaherrera, W. (2021). Covid- 19: La transformación de la educación en el Ecuador mediante la inclusión de herramientas tecnológicas en las clases virtuales. Revista Científica Dominio de las Ciencias 7 (1). https://dominiodelasciencias.com/ojs/index.php/es/article/view/1684

Pérez, A. (2020). Competencia digital docente para la reducción de la brecha digital: Estudio comparativo de España y Costa Rica. Revista Tripodos, 46. http://www.tripodos.com/index.php/Facultat_Comunicacio_Blanquerna/article/view/790

Schenke, E. \& Pérez, M. I. (2018). Un abordaje teórico de la investigación cualitativa como enfoque metodológico. Revista Acta Geográfica $12 \quad$ (30). https://revista.ufrr.br/actageo/article/view/5201/2603

Sucari, W. (2019). Participación familiar en la educación escolar Perú. Universidad Nacional del Altiplano y Universidad Nacional Mayor de San Marcos. Revista Innova Educación 1 (1) http://www.revistainnovaeducacion.com/index.php/rie/article/view/1/3 